\title{
Biomaterials
}

\section{Assessment and modeling of poly(vinyl alcohol) bioartificial pancreas in vivo}

\author{
Tai-Horng Young ${ }^{\mathrm{a}, *}$, Wen-Yuan Chuang ${ }^{\mathrm{a}}$, Meng-Ying Hsieh ${ }^{\mathrm{b}}$, Leo-Wang Chen ${ }^{\mathrm{b}}$, \\ Jyh-Ping $\mathrm{Hsu}^{\mathrm{b}}$ \\ ${ }^{a}$ Institute of Biomedical Engineering, College of Medicine and College of Engineering, National Taiwan University, Taipei 10016, Taiwan, ROC \\ ${ }^{\mathrm{b}}$ Department of Chemical Engineering, National Taiwan University, Taipei 10617, Taiwan, ROC
}

Received 6 February 2001; accepted 20 February 2002

\begin{abstract}
Pancreatic islets surrounded by a semipermeable membrane to prevent an immune response by the host immunosystem is a potential way of treating type I diabetes mellitus. Our previous in vitro studies have demonstrated that poly(vinyl alcohol) (PVA) membranes satisfy the basic requirements for a bioartificial pancreas. This study was designed to evaluate the performance of PVA tubular membrane chambers containing islets in vivo. When the $\mathrm{m}-2$ type of PVA chamber was implanted into streptozotocininduced diabetic rats, nonfasting blood glucose levels dropped from $500 \pm 35 \mathrm{mg} / \mathrm{dl}$ to the lowest value $210 \pm 22 \mathrm{mg} / \mathrm{dl}$. Furthermore, the performance of the bioartificial pancreas can be enhanced by the increased numbers of implanted chambers. If three $\mathrm{m}-2$ chambers were implanted, nonfasting blood glucose levels in the diabetic rats decreased to $130-160 \mathrm{mg} / \mathrm{dl}$ and such a low blood glucose value was maintained for 1 month. This indicates that implanting three $\mathrm{m}-2$ chambers in the diabetic rats could provide improved permeability of insulin to normalize blood glucose levels and improved survival of islets from the immune system of the recipient. For improving the design of the bioartificial pancreas, a mathematical model was developed to account for the changes in blood glucose levels of the diabetic rats. We demonstrated such a mathematical analysis was helpful to understand the characteristics of islet inside an artificial environment. (C) 2002 Elsevier Science Ltd. All rights reserved.
\end{abstract}

Keywords: PVA tubular membrane chambers; Bioartificial pancreas; Diabetes; Mathematical model

\section{Introduction}

Long-term blood glucose normalization in diabetes mellitus is an important goal. The use of conventional insulin therapy for control of glucose levels continues to develop serious vascular and neurological complications in diabetic patients. Therefore, much effort has focused on the development of the bioartificial pancreas, in which the transplanted pancreatic islet tissue is enclosed within a semipermeable membrane, for treatment of human diabetes [1-11]. The semipermeable membrane is permeable to small molecules such as nutrients, electrolytes, oxygen, and bioactive secretory products but totally impermeable to large molecules such as antibodies and lymphocytes. Such an immunoisolation

\footnotetext{
*Corresponding author. Tel.: +886-2-23123456 ext. 1455; fax: + 886-2-23940049.

E-mail address: thyoung@ha.mc.ntu.edu.tw (T.-H. Young).
}

technique can transplant islets without any immunosuppressive therapy, thus eliminating the need for immunosuppressive drugs and allowing the use of nonhuman donor islets.

Our previous in vitro studies have demonstrated that poly(vinyl alcohol) (PVA) membranes satisfy the basic requirements for a bioartificial pancreas: good permeability for glucose, insulin and albumin but the passage of immunoglobulin $\mathrm{G}$ was completely prevented [8]. In addition, islets cultured in the PVA tubular membranes could perform their function of secreting insulin after 30 days in the static incubation study and rapidly releasing insulin through the membranes in response to changes in concentrations of glucose in the dynamic perifusion experiment [9]. In this work, data obtained from in vivo transplantation studies supported the previous assessment that islets entrapped by the PVA tubular membrane chamber could control the glucose level in diabetic rats. Therefore, this membrane provided 
adequate performance for secretory products in an application as a synthetic extracellular matrix for a bioartificial pancreas. Furthermore, a mathematical model, which evaluated the performance of a bioartificial pancreas, was proposed and compared with the in vivo data. We demonstrated such a mathematical analysis was helpful to understand the characteristics of islet inside an artificial environment.

\section{Materials and methods}

\subsection{Chamber preparation}

Two (m-1 and m-2) PVA (molecular weight 74,800, Chang Chun Co., Taiwan) tubular membrane chambers were constructed according to the procedure described previously [9]. Briefly, PVA solution (14 wt \% in water) was coated on a glass bar (diameter: $2 \mathrm{~mm}$ ) by a dipping method and then immersed into a coagulation bath with $\mathrm{Na}_{2} \mathrm{SO}_{4} / \mathrm{KOH} / \mathrm{H}_{2} \mathrm{O}=$ saturated $/ 75 \mathrm{~g} / 1000 \mathrm{ml}$ to obtain a tubular membrane with a uniform thickness of about $100 \mu \mathrm{m}$. Basically, m-1 and m-2 chambers were identical except for their outer surface wall; see Fig. 1 in Ref. [9]. M-1 chambers had a totally dense skin layer, whereas m2 chambers were prepared with added polyethylene glycol into PVA solution to create pores in the surface to improve their permeability [9].

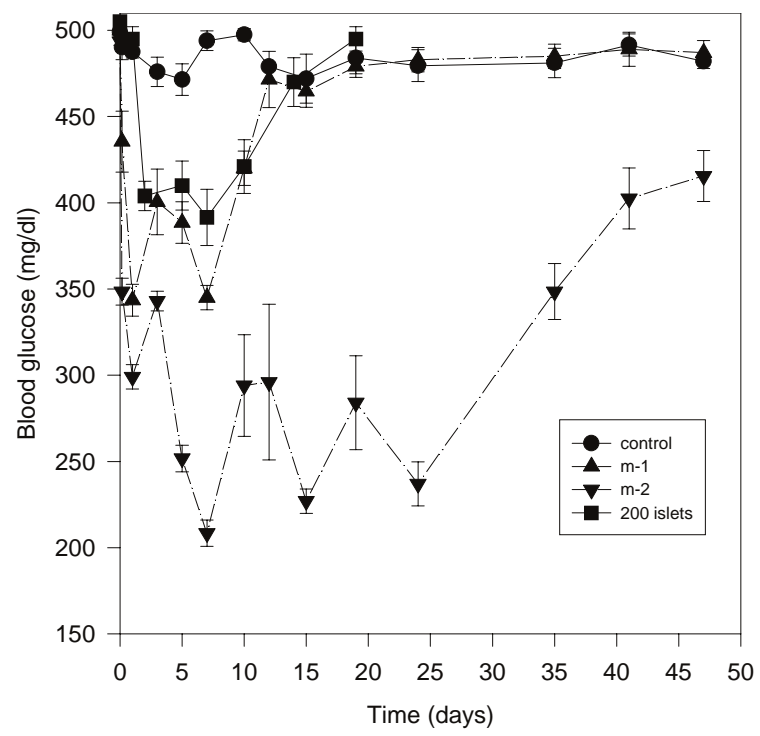

Fig. 1. Nonfasting blood glucose levels of diabetic rats, diabetic rats transplanted with 200 free islets, and diabetic rats transplanted with 80 islets seeded in the m-1 and m-2 tubular membrane chamber. For each case, three diabetic rats underwent operation. Data are presented as the mean \pm standard deviation. Nonfasting blood glucose levels of diabetic rats transplanted with 80 islets seeded in the $\mathrm{m}-2$ tubular membrane chamber denote significant differences compared to the diabetic rats without any peritoneal transplantation during the total implantation period $(p<0.05)$.

\subsection{Islet isolation and chamber seeding}

Rat islets were isolated from male Wistar rats (250$350 \mathrm{~g}$ ) by the collagenase technique, separated on a Ficoll gradient, and hand-picked with the aid of a dissecting microscope to count the number of islets following the method of Lacy and Kostianovsky [12]. Islets were cultured in RPMI-1640 medium supplemented with $10 \%$ fetal bovine serum and $1 \%$ antibiotics in a humidified atmosphere of $95 \%$ air $/ 5 \% \mathrm{CO}_{2}$ at $37^{\circ} \mathrm{C}$ for 2 days and then seeded into $4 \mathrm{~cm} \mathrm{~m}-1$ and $\mathrm{m}-2$ chambers Each chamber was seeded with about 80 islets. The open ends of the chamber were sealed with polytetrafluoroethylene plugs as previously described [9].

\subsection{Chamber implantation}

Diabetes was induced in Wistar rats (250-350 g) by an intravenous injection of streptozotocin (STZ) into the tail vein $(60 \mathrm{mg})$ about 10 days prior to implantation. Nonfasting blood glucose concentrations were measured by tail bleeding using a glucose analyzer. Only rats with blood glucose level $>400 \mathrm{mg} / \mathrm{dl}$ were used in this study.

PVA chamber seeded with islets was implanted into the peritoneal cavity of STZ-induced diabetic rats. Diabetic rats were anaesthetized with an intramuscular injection of a mixture of ketamine $(35 \mathrm{mg} / \mathrm{kg})$ and xylazine $(5 \mathrm{mg} / \mathrm{kg})$, and prepared for surgery by shaving their abdomens and then scrubbing with alcohol solution. After surgery, changes in nonfasting blood glucose levels in the diabetic rats were measured about two or three times weekly. In addition to evaluation of the effect of single chamber seeded with islets, more m-2 chambers were implanted in the diabetic rats to improve the control of blood glucose levels due to $\mathrm{m}-2$ chamber membrane with better permeability than m-1 [9]. Furthermore, for comparison, changes in nonfasting blood glucose levels in diabetic rats without implantation and with approximately 200 free islets (not seeded in PVA tubular membrane chamber) implanted into the peritoneal cavity were observed in our study.

For each case, three diabetic rats underwent operation. Blood glucose levels were expressed as mean \pm SEM. Statistical significance was calculated using one way analysis of variance (ANOVA) followed by Student's $t$-test $(p<0.05$ was considered significant).

\subsection{Mathematical model}

For improving the design of a bioartificial pancreas, a mathematical model was developed to account for the changes in blood glucose levels of the diabetic rats. The theoretical analysis describing the initially rapid decrease of blood glucose concentration, maintaining a constant level and the finally rapid rise of blood glucose concentration was derived as follows. Let $N$ be the 
number of islets placed in a chamber, and $C$ be the blood glucose concentration of a rat. Initially, 80 islets were seeded in each chamber. In the previous perifusion study [9], the contribution of most islets in the chamber was insignificant. Due to the fact that only some of the islets functioning to secrete insulin, we assume that there exists a critical number of islets, $N_{\mathrm{c}}$. If $N>N_{\mathrm{c}}$, the number of effective (working) islets becomes $N_{\mathrm{c}}$. In this case, the rate of decrease in the blood glucose concentration is proportional to $N_{\mathrm{c}}$. Also, suppose that the blood glucose concentration has a lower bound, $C_{\mathrm{k}}$, which can be estimated on the basis of experimental data. That is, the blood glucose concentration cannot be lower than a minimum level. On the other hand, if $N<N_{\mathrm{c}}$, the number of effective islets is $N$, and the blood glucose concentration will increase with time, and we assume that the rate of increase is proportional to $\left(N_{\mathrm{c}}-N\right)$. Concerning the decrease in the number of islets, a reasonable result is most likely preceded by a progressive decrease in B-cell mass. Either of the aggregation of the islets inside the chamber $[3,4,13,14]$, the PVA chamber encapsulated by connective tissue, or the permeability of small molecules like cytokines, which are toxic to islets $[15,16]$, could contribute to decrease viability of islet cells to result in cell death by necrosis or apoptosis. Here, we assume that each islet has the same probability of mortality, thus, the higher number of islets will lead to the higher number of deaths at a fixed period, with the result that the number of islets decreases with time and the rate of decrease in the number of islets is proportional to $N$. Based upon the above discussions, the temporal variations in the blood glucose concentration and the number of islets can be described by

$-\frac{\mathrm{d} C}{\mathrm{~d} t}=k_{1} N_{\mathrm{c}}, \quad N>N_{\mathrm{c}}$,

$C=C_{\mathrm{k}}, \quad C<C_{\mathrm{k}}$,

$\frac{\mathrm{d} C}{\mathrm{~d} t}=k_{2}\left(N_{\mathrm{c}}-N\right), \quad N<N_{\mathrm{c}}$,

$\frac{\mathrm{d} N}{\mathrm{~d} t}=-k_{3} N$.

Here, $t$ denotes time and $k_{1}, k_{2}$, and $k_{3}$ are rate constants, which are related to the characteristics of an artificial pancreas.

\section{Results and discussion}

Nonfasting blood glucose levels of diabetic rats, diabetic rats transplanted with 200 free islets, and diabetic rats transplanted with 80 islets seeded in the $\mathrm{m}-1$ and $\mathrm{m}-2$ chambers are shown in Fig. 1 . In the diabetic rats without any peritoneal transplantation, nonfasting blood glucose levels showed a hyperglycemic state (about $460-500 \mathrm{mg} / \mathrm{dl}$ ) and no significant changes. In the three diabetic rats receiving m-1 chamber with 80 islets, nonfasting blood glucose levels dropped from $500 \pm 35 \mathrm{mg} / \mathrm{dl}$ to the lowest value $340 \pm 20 \mathrm{mg} / \mathrm{dl}$. The in vivo performance of the $\mathrm{m}-1$ chamber was not good. It could not reverse the hyperglycemic state and it only maintained insulin secretion activity for 10 days. In contrast, nonfasting blood glucose levels in recipient diabetic rats were significantly decreased after the intraperitoneal transplantation of the m-2 chamber seeded with 80 islets compared to the diabetic rats without any peritoneal transplantation during the period of transplantation $(p<0.05)$. The lowest value obtained was about $210 \pm 22 \mathrm{mg} / \mathrm{dl}$, close to a reasonable definition for a successful artificial pancreas that achieves a blood glucose level $<200 / \mathrm{mg} / \mathrm{dl}$ [6]. Rats sustained nonfasting blood glucose levels $<300 \mathrm{mg} / \mathrm{dl}$ for about 20 days. On the contrary, free islets transplants showed slight decreases in nonfasting blood glucose levels for only 7 days. This indicates that the $\mathrm{m}-2$ chamber could provide improved permeability of insulin to normalize blood glucose levels and improved protection of islets from the immune system of the recipient.

Since intraperitoneal transplants of the $\mathrm{m}-2$ chamber reversed the hyperglycemic state, we implanted more m2 chambers in the diabetic rats to check whether blood glucose levels could be decreased to a lower value and sustained for a longer period. Fig. 2 shows comparative study on changes in nonfasting blood glucose levels of diabetic rats after intraperitoneal transplantation of one, two and three m-2 chambers. Each implanted chamber

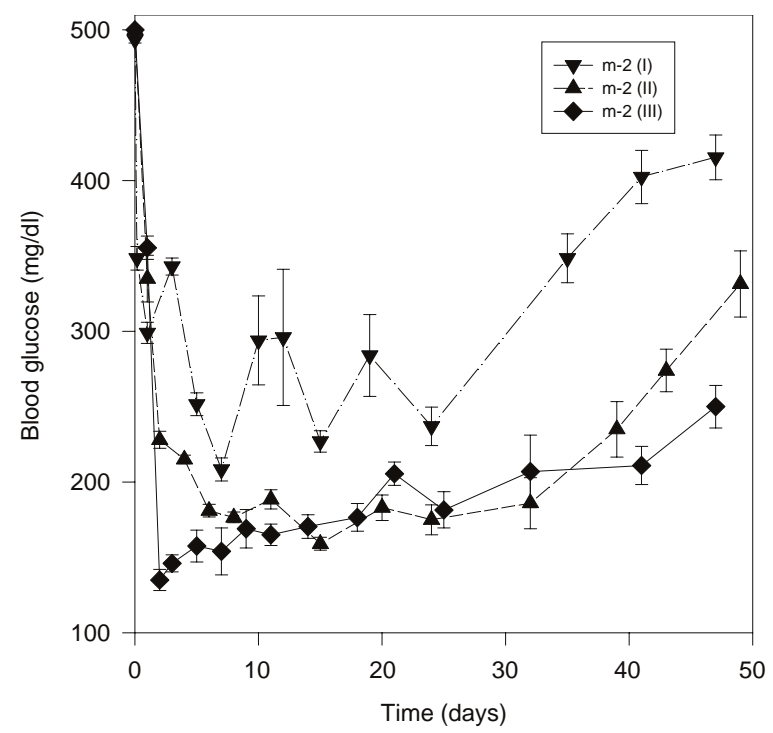

Fig. 2. Comparative study on changes in nonfasting blood glucose levels among diabetic rats transplanted with one, two and three $\mathrm{m}-2$ chambers. (The letters in parentheses represent the number of implanted m-2 chambers.) Each chamber contains 80 islets. For each case, three diabetic rats underwent operation. Data are presented as the mean \pm standard deviation. 

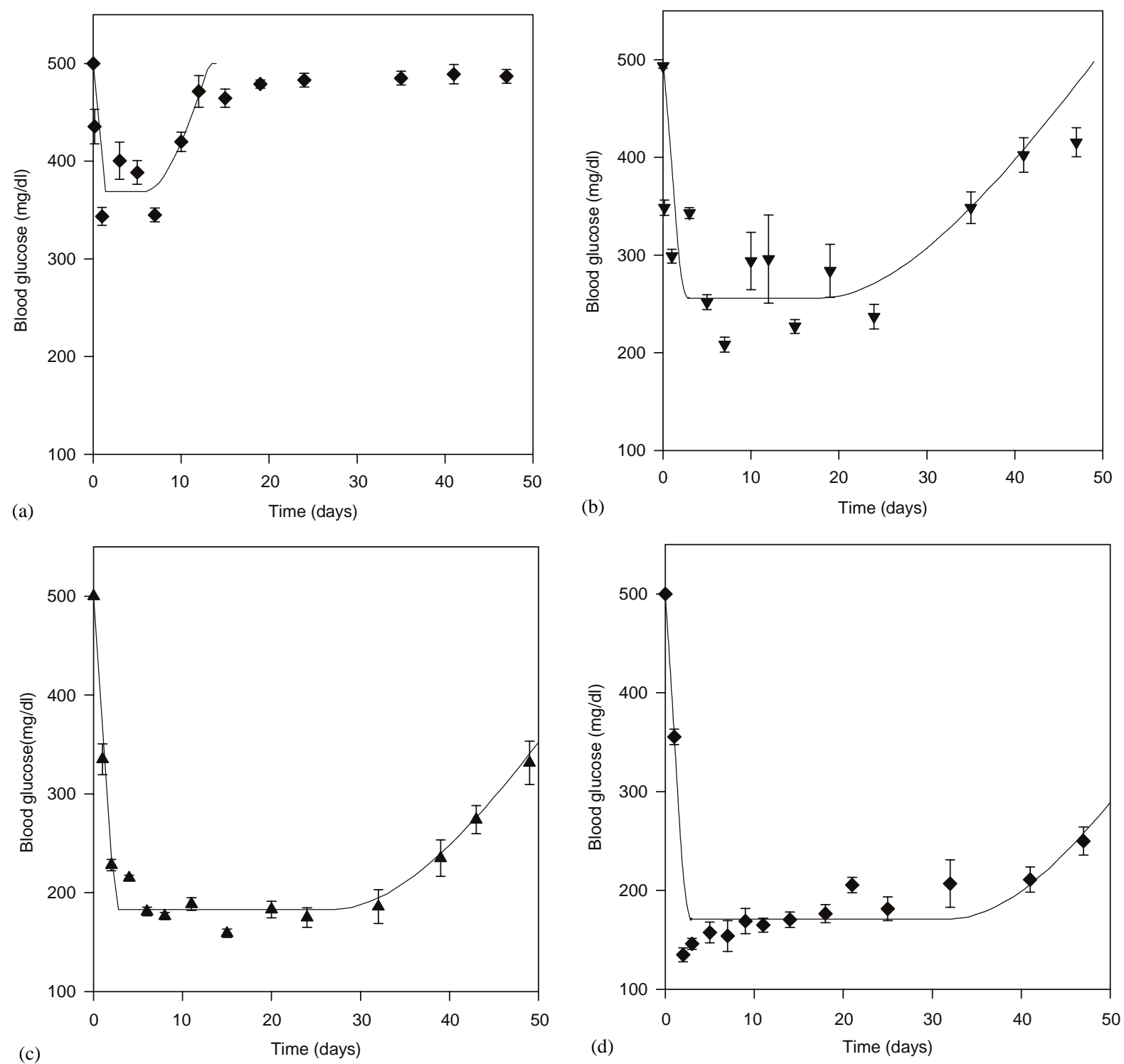

Fig. 3. The comparison in blood glucose levels between the experimental data and the theoretical prediction. The parameters used in fitting the experimental data are given in Table 1. (a) $\mathrm{m}-1$; (b) $\mathrm{m}-2$ (I); (c) $\mathrm{m}-2$ (II) and (d) $\mathrm{m}-2$ (III). (The letters in parentheses represent the number of implanted m-2 chambers.)

contained 80 islets. Clearly, blood glucose normalization in diabetes mellitus became better when the number of implanted $\mathrm{m}-2$ chambers increased. Nonfasting blood glucose levels in the diabetic rats receiving intraperitoneal transplantation of three $\mathrm{m}-2$ tubular membrane chambers decreased from pretransplant level of 460-500 to $130-160 \mathrm{mg} / \mathrm{dl}$ and such a low blood glucose value was maintained for 1 month following intraperitoneal transplantation. This demonstrated the successful functioning of the m-2 chamber seeded with islets as a bioartificial pancreas.

It is reasonable to attribute the lower blood glucose levels to more islets in three $\mathrm{m}-2$ chambers. However, 200 free islets transplants did not show significant contribution in decreasing blood glucose levels. Especially, three m-2 chambers can prolong the function time of the artificial pancreas. Therefore, the mathematical model mentioned above was used to evaluate the performance of m-1 and m-2 PVA chambers used in the artificial pancreas.

Based on our model, the change in blood glucose levels of the diabetic rats is divided into three stages: rapid decrease, constant low level and rapid rise of blood glucose concentration. Fig. 3 shows the model can appropriately fit the variation of blood glucose level for transplantation of the m-1 chamber and for transplantation of one, two and three $\mathrm{m}-2$ chambers. In the simulation, the best fitted parameters, $k_{1}, k_{2}, k_{3}$ and $N_{\mathrm{c}}$ were estimated and summarized in Table 1 . Regardless of the number of the m-2 chambers implanted in the rats, the $k_{1}, k_{2}$ and $k_{3}$ values do not have significant difference, however, these three parameters have 
significant difference between the m-1 and m-2 chambers. The rate constant $k_{1}$ in Eq. (1) mainly depends upon the permeation rate of insulin secreted by islets through the membrane, which is influenced by the skin layer structure of the membrane $[8,9]$. The larger the value of $k_{1}$ the better the performance of a device. As can be seen in Table 1, the performance of the $m-2$ chamber is better than that of the $\mathrm{m}-1$ chamber. This result is consistent with the effect of adding polyethylene glycol to the $\mathrm{m}-2$ chamber to create pores in the skin layer, leading to increased membrane permeability. In addition, the definition of $N_{\mathrm{c}}$, given in Eq. (1), suggests that the larger its value the more insulin secreted by effective islets, and the better the performance of a device. According to Table 1, the performance of the devices examined, from the best to the worst, follows the order $\mathrm{m}-2$ (III) $>\mathrm{m}-2$ (II) $>\mathrm{m}-2$ (I) $>\mathrm{m}-1$. (The letters in parentheses represent the number of implanted $\mathrm{m}-2$ chambers.) Combining the effect of $k_{1}$ with $N_{\mathrm{c}}$, the

Table 1

Summary of the estimated parameters in fitting the experimental data shown in Fig. 3

\begin{tabular}{lllll}
\hline & $\mathrm{m}-1$ & $\mathrm{~m}-2(\mathrm{I})^{\mathrm{a}}$ & $\mathrm{m}-2(\mathrm{II})^{\mathrm{a}}$ & $\mathrm{m}-2(\mathrm{III})^{\mathrm{a}}$ \\
\hline$K_{1}$ & 4.2 & 4.5 & 4.7 & 4.7 \\
$K_{2}$ & 1.6 & 0.56 & 0.54 & 0.53 \\
$K_{3}$ & 0.22 & 0.067 & 0.065 & 0.065 \\
$N_{\mathrm{c}}$ & 22 & 24 & 28 & 30 \\
& & & $(14,14)^{\mathrm{b}}$ & $(10,10,10)^{\mathrm{b}}$ \\
$T_{\mathrm{o}}$ & 4.5 & 16 & 24 & 30 \\
$C_{\mathrm{k}}$ & 369 & 256 & 183 & 173 \\
\hline
\end{tabular}

${ }^{\mathrm{a}}$ I, II, and III refer to the number of implanted $\mathrm{m}-2$ chambers.

${ }^{\mathrm{b}}$ The critical number of islets in each implanted $\mathrm{m}-2$ chamber. performance of the devices examined, from the best to the worst, follows the order $\mathrm{m}-2$ (III) $>\mathrm{m}-2$ (II) $>\mathrm{m}$ 2(I) $>\mathrm{m}-1$.

The definition of $C_{\mathrm{k}}$, estimated by a linear regression analysis on the experimental blood glucose concentrations, implies that the lower its level the better the performance of a device. The level of $C_{\mathrm{k}}$ for each device follows the order $\mathrm{m}-2$ (III) $<\mathrm{m}-2$ (II) $<\mathrm{m}-2$ (I) $<\mathrm{m}-1$, as indicated in Table 1. That is, the performance of the devices examined, from the best to the worst, follows the order $\mathrm{m}-2$ (III) $>\mathrm{m}-2$ (II) $>\mathrm{m}-2$ (I) $>\mathrm{m}-1$, which is consistent with the combined effect of $k_{1}$ and $N_{\mathrm{c}}$. Theoretically, the greater the value of $k_{1}$ multiplied by $N_{\mathrm{c}}$, the lower the value of $C_{\mathrm{k}}$. Fig. 4 shows the level of $C_{\mathrm{k}}$ seems to have a limiting value about $170 \mathrm{mg} / \mathrm{dl}$ as the value of $k_{1}$ multiplied by $N_{\mathrm{c}}$ (or the number of implanted $\mathrm{m}-2$ chambers) increases. Therefore, implanting three $\mathrm{m}-2$ chambers in the diabetic rats is good enough for glycemic normalization.

Eq. (3) suggests that the larger the value $k_{2}$, the faster the rate of increase in blood glucose concentration, and the worse the performance of a device. According to Table 1 , the $k_{2}$ value of the m-1 chamber is greater than that of the m-2 chamber. It is reasonable that $k_{1}$ and $k_{2}$ have the opposite tendency from the view point of the membrane permeability.

Eq. (4) shows that $k_{3}$ is related to the rate of decrease of the number of islets; the smaller its value the lower the rate of decrease of the number of islets, and the better the performance of a device. Table 1 implies that the $k_{3}$ value of the m-2 chamber is less than that of the $\mathrm{m}-1$ chamber. Another parameter describing the effect of the $k_{3}$ is the time interval, $T_{\mathrm{o}}$, in which the blood

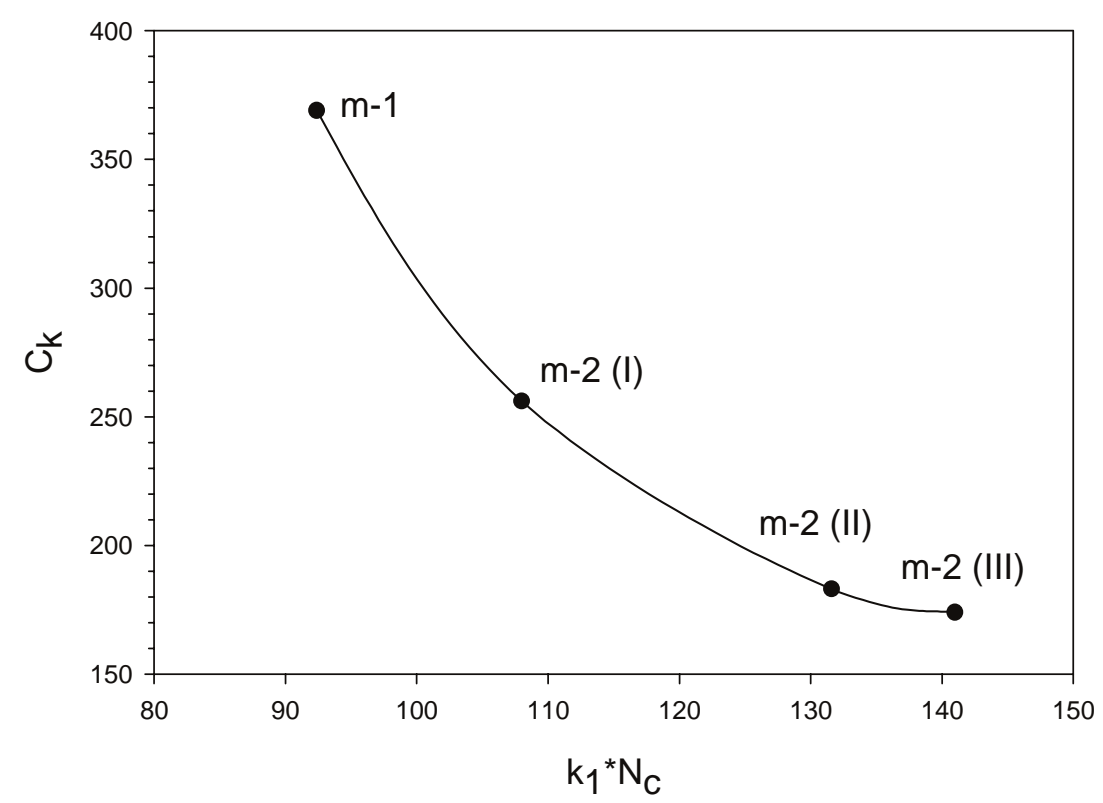

Fig. 4. The relation between the level of $C_{\mathrm{k}}$ and the value of $k_{1}$ multiplied by $N_{\mathrm{c}}$ for different devices. (The letters in parentheses represent the number of implanted $\mathrm{m}-2$ chambers.) 
glucose concentration can be maintained at a certain low level. When the number of "viable" islets was less than $N_{\mathrm{c}}$, the level of blood glucose concentration is rapidly elevated. Apparently, the larger the $T_{\mathrm{o}}$ the better the performance of a device. Table 1 reveals that the performance of the devices examined, from the best to the worst follows the order $\mathrm{m}-2$ (III) $>\mathrm{m}-2$ (II) $>\mathrm{m}-2$ (I). Thus, the survival of islets was better for multiplechamber implantation than single-chamber implantation. Prolonged survival of islets is probably related to the diminished excessive demands on its function from persisting stimulus of high blood glucose levels. In addition, Table 1 shows each chamber had smaller value for $N_{\mathrm{c}}$ when the number of implanted chambers increased. This also suggests the multiple-chamber implantation can satisfy the requirement of $N>N_{\mathrm{c}}$ for a longer period.

At this point it is evident that the m-2 chamber in the diabetic rats could provide improved permeability of insulin to normalize blood glucose levels and improved survival of islets from the immune system of the recipient and its performance could be enhanced by the increased number of implanted chambers. Two questions about $N_{\mathrm{c}}$, however, remained to be answered. First, what is the mechanism of the effect of m-1 and m2 chambers on $N_{\mathrm{c}}$ ? It has been suggested by Bergen et al. [17] that insulin secretion by rat islets placed in an artificial pancreas was inhibited by the insulin existing inside the device. They recommended that this inhibition phenomenon should be considered in the design of a transplantation chamber. In fact, the diffusion rate of glucose and the insulin secretion rate by islets are relatively rapid compared to the diffusion rate of insulin [8], so insulin will accumulate in a chamber. Therefore, even if the insulin concentration inside the chamber will increase to create an insulin concentration gradient across the chamber, the elevated insulin concentration may inhibit insulin secretion by islets. The outcome is comparable to the number of working islets less than the actual number of islets placed in the chamber. Thus, the mathematical function of a critical number of islets, $N_{\mathrm{c}}$ was introduced to our model to account for the inhibition mechanism. The results of simulation show 22 and 24 islets functioned to secrete insulin to regulate the blood glucose level for the $\mathrm{m}-1$ and $\mathrm{m}-2$ chambers, respectively. This can be attributed to that the insulin concentration inside the m- 1 chamber will accumulate to a higher value owing to its lower insulin diffusion rate $[8,9]$, which is responsible for the smaller $N_{\mathrm{c}}$. Secondly, what is the mechanism of the effect of the number of implanted $\mathrm{m}-2$ chambers on $N_{\mathrm{c}}$ ? When the number of implanted chambers is increased, the observed decrease in $N_{\mathrm{c}}$ of each implanted chamber can be interpreted by the insulin needed from each chamber for glycemic normalization is reduced, leading to a less islets functioning to secrete insulin. Due to the limited amount of data it is not clear if this interpretation is realistic. However, it is reasonable that the larger number of total working islets is needed for lower $C_{\mathrm{k}}$ when the number of implanted chambers increases. In summary, we conclude that the performance of $\mathrm{m}$-2(III) is better than that of the other three types of devices.

\section{Acknowledgements}

The authors thank the National Science Council of Taiwan, the Republic of China for their financial support of this research.

\section{References}

[1] Lim F, Sun AM. Microencapsulated islets as bioartificial endocrine pancreas. Science 1980;210:908-10.

[2] Altman JJ, Houlbert A, Callard P, McMillan P, Solomon BA, Rosen J, Galetti PM. Long term plasma glucose normalization in experimental diabetic rats with macroencapsulated implants of benign human insulinomas. Diabetes 1986;35:625-33.

[3] Sullivan SJ, Maki T, Borland KM, Mahoney MD, Solomon BA, Muller TE, Monaco AP, Chick WL. Biohybrid artificial pancreas: long-term implantation studies in diabetic, pancreatectomized dogs. Science 1991;252:718-21.

[4] Lacy PE, Hegre OD, Vazeou AG, Gentile FT, Dionne KE. Maintenance of normoglycemia in diabetic mice by subcutaneous xenograft of encapsulated islets. Science 1991;254:1782-4.

[5] Lanza RP, Beyer AM, Chick WL. Xenogenic humoral responses to islets transplanted in biohybrid diffusion chambers. Transplantation 1994;57:1371-5.

[6] Inoue K, Fujisato T, Gu YJ, Burczak K, Sumi S, Kogire M, Tobe T, Uchida K, Nakai I, Maetani S, Ikada Y. Experimental hybrid islet transplantation: application of polyvinyl membrane for entrapment islets. Pancreas 1992;7:562-8.

[7] Kessler L, Legeay G, Jesser C, Damge C, Pinget M. Influence of corona surface treatment on the properties of an artificial membrane used for Langerhans islets encapsulation: permeability and biocompatibility studies. Biomaterials 1995;16:185-91.

[8] Young TH, Yao NK, Chang RF, Chen LW. Evaluation of asymmetric poly(vinyl alcohol) membranes for use in the artificial islets. Biomaterials 1996;17:2131-7.

[9] Young TH, Yao NK, Chuang WY, Chen LW. Use of a diffusion model for assessing the performance of poly(vinyl alcohol) bioartificial pancreases. J Biomed Mater Res 1998;40:385-91.

[10] Ohgawara H, Hirotani S, Miyazaki J, Teraoka S. Membrane immunoisolation of a diffusion chamber for bioartificial pancreas. Artif Organs 1998;22:788-94.

[11] Hirotani S, Eda R, Kawabata T, Fuchinoue S, Teraoka S, Agishi T, Ohgawara H. Bioartificial endocrine pancreas (Bio-AEP) for treatment of diabetes: effect of implantation of Bio-AEP on the pancreas. Cell Transplant 1999;8:399-404.

[12] Lacy PE, Kostianovski M. Method for the isolation of intact islets of Langerhans from the rat pancreas. Diabetes 1967;16:35-9.

[13] Aung T, Kogire M, Inoue K, Fujisato T, Gu Y, Burczak K, Shinohara S, Mitsuo M, Maetani S, Ikada Y, Tobe T. Insulin release from a bioartificial pancreas using a mesh reinforced polyvinyl alcohol hydrogel tube. ASAIO J 1993;39:93-6.

[14] Aung T, Inoue K, Kogire M, Doi R, Kaji H, Tun T, Hayashi H, Echigo $\mathrm{Y}$, Wada $\mathrm{M}$, Imamura $\mathrm{M}$, Fujisato $\mathrm{T}$, Maetani $\mathrm{S}$, Iwata H, Ikada Y. Comparison of various gels for immobilization 
of islets in bioartificial pancreas using a mesh-reinforced polyvinyl alcohol hydrogel tube. Transplantat Proc 1995;27:619-21.

[15] Delaney CA, Pavlovic D, Hoorens A, Pipeleers DG, Eizirik. Cytkines induces deoxyribonucleic acid strand breaks and apoptosis in human pancreatic islet cells. Endocrinology 1997; 138:2610-4.
[16] Suarez-Pinzon WL, Strynadka K, Rabinovitch A. Destruction of rat pancreatic islet $\beta$-cells by cytokines involves the production of cytotoxic aldehydes. Endocrinology 1996;137:5290-6.

[17] Bergen JF, Mason NS, Scharp DW, Sparks RE. Insulin inhibition of islets in transplantation chambers. Artif Organs 1978;172: $144-50$. 